\title{
High Signal Intensity in Arterial Walls on Diffusion-Weighted Magnetic Resonance Imaging in the Active Phase of Takayasu Arteritis
}

Yasuyoshi Kuroiwa, PhD; Hiroshi Tai, MD; Atsushi Yamashita, MD, PhD;

Eiji Furukoji, MD, PhD; Yasushi Kihara, MD; Toshinori Hirai, MD, PhD;

Yujiro Asada, MD, PhD; Takuroh Imamura, MD, PhD

A

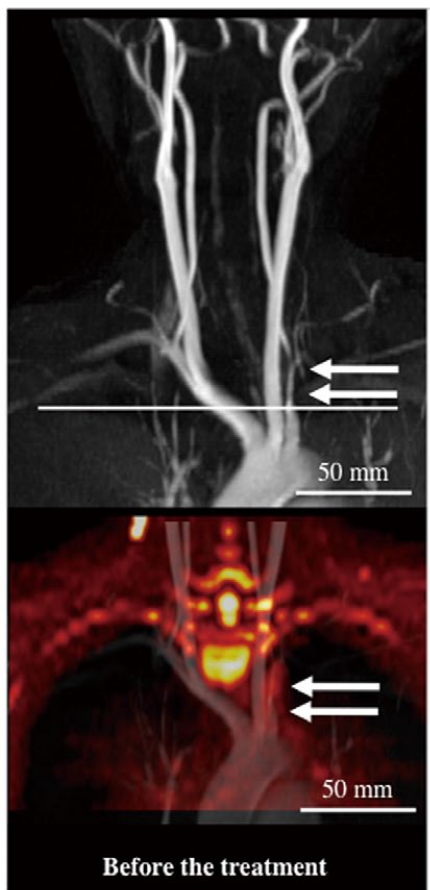

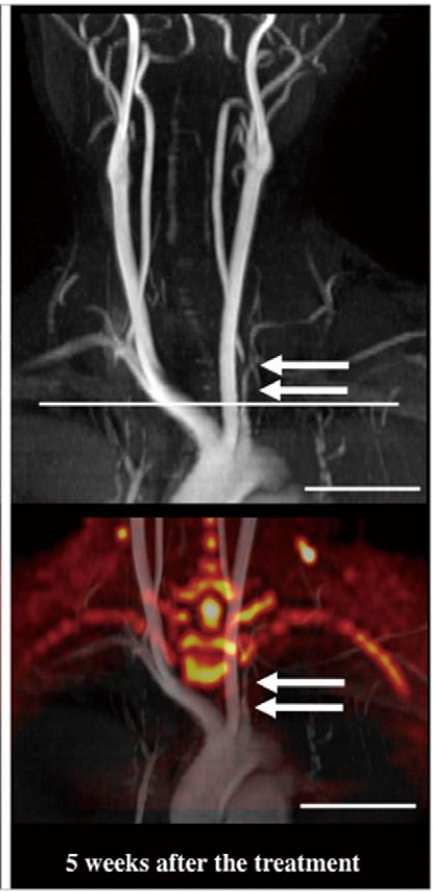

5 weeks after the treatment
B

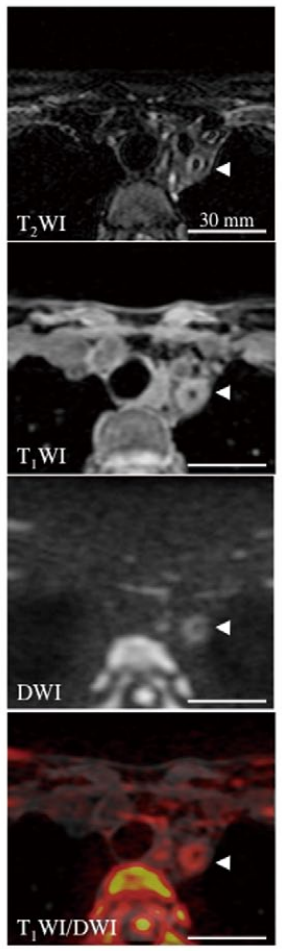

Before the treatment

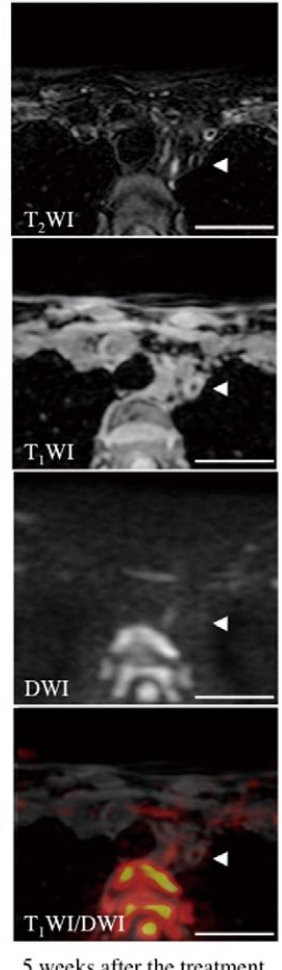

5 weeks after the treatment

Figure. (A) Maximum intensity projection (MIP) of cardiac-gated time-of-flight magnetic resonance angiography (MRA) showing luminal stenosis of the left subclavian artery before and after 5 weeks of oral steroid treatment (arrows). Coronal MIP of the diffusion-weighted imaging (DWI) fused with MRA shows high signal intensity in the left subclavian artery before treatment, and disappearance of the arterial high signal intensity after 5 weeks of treatment. The fused images also show high signal intensity in the bone marrow of vertebra and ribs. White horizontal line, (B) level of the axial imaging. (B) Axial $T_{2}$ - and $T_{1}$-weighted imaging ( $T_{2} W I$, $\mathrm{T}_{1} \mathrm{WI}$ ) with fat suppression showing marked thickening of the arterial wall with luminal stenosis. DWI and fusion of DWI and $\mathrm{T}_{1} \mathrm{Wl}$ show high signal intensity in the thickened wall of the left subclavian artery. After 5 weeks of oral steroid treatment, axial $\mathrm{T}_{2} \mathrm{Wl}$ and TiWI with fat suppression show reduction of arterial swelling and disappearance of the arterial high signal intensity on DWI and merged DWI-TiWI.

Received January 27, 2017; revised manuscript received March 25, 2017; accepted March 28, 2017; released online April 13, 2017 Time for primary review: 19 days

Department of Radiological Technology (Y. Kuroiwa), Department of Internal Medicine (H.T., T.I.), Department of Radiology (Y. Kihara), Koga General Hospital, Miyazaki; Department of Pathology (Y. Kuroiwa, A.Y., Y.A.), Department of Radiology (E.F., T.H.), Faculty of Medicine, University of Miyazaki, Miyazaki, Japan.

Mailing address: Atsushi Yamashita, MD, PhD, Department of Pathology, Faculty of Medicine, University of Miyazaki, 5200 Kihara, Kiyotake, Miyazaki 889-1692 Japan. E-mail: atsushi_yamashita@med.miyazaki-u.ac.jp

ISSN-1346-9843 All rights are reserved to the Japanese Circulation Society. For permissions, please e-mail: cj@j-circ.or.jp 
$\mathbf{T}$ akayasu arteritis is a granulomatous arteritis that can affect the aorta and its major branches, and is characterized by visual disturbances and marked weakening of the pulses in the upper extremities. The systemic inflammatory activity of the disease is assessed using erythrocyte sedimentation rate and serum C-reactive protein (CRP). The regional inflammatory activity of the arteritis is currently assessed on ultrasonography, ${ }^{18} \mathrm{~F}$-fluorodeoxyglucose (FDG) uptake on positron emission tomography (PET)/computed tomography (CT), ${ }^{\mathbf{1}}$ and delayed gadolinium enhancement on magnetic resonance imaging (MRI). ${ }^{2}$ Increased ${ }^{18} \mathrm{~F}-\mathrm{FDG}$ uptake by macrophages on PET/CT occurs in sites of arterial inflammation; and delayed gadolinium enhancement on $\mathrm{T}_{1}$-weighted MRI is associated with edema of the arterial wall caused by inflammation. Diffusion-weighted imaging (DWI) is an MRI methodology that measures the diffusion of water molecules, and can be used to detect the acute phases of inflammation and cerebral infarction. DWI, image fusion, and apparent diffusion coefficient (ADC) data, however, have not been reported in Takayasu arteritis. We applied this technique to evaluate regional inflammatory activity in Takayasu arteritis.

A 29-year-old woman with ulcerative colitis was admitted with a 5-month history of headache, transient visual disturbance, weakness and coldness of the left upper extremity, left-sided abdominal pain, and dry cough. Physical examination indicated pulselessness and unmeasurable blood pressure in the left radial artery (blood pressure in the right radial artery, $106 / 63 \mathrm{mmHg}$ ). Laboratory results indicated elevated serum CRP $(7.2 \mathrm{mg} / \mathrm{dL})$. To evaluate the arteries, we performed non-contrast cardiac gated 2-D time-of-flight MR angiography (MRA) using maximum intensity projections (MIP), and $\mathrm{T}_{2}, \mathrm{~T}_{1-}$, and diffusion-weighted MRI (b values, 0 and $1,000 \mathrm{~mm}^{2} / \mathrm{s}$; Signa HDxt 1.5T, GE Healthcare). Coronal MIP of the MRA and merged MRA and DWI indicated luminal stenosis with high signal intensity on the left subclavian artery (Figure A). Axial $\mathrm{T}_{2}$ - and $\mathrm{T}_{1}$-weighted imaging $\left(\mathrm{T}_{2} \mathrm{WI}\right.$, $\mathrm{T}_{1} \mathrm{WI}$ ) with fat suppression showed marked thickening of the arterial wall with luminal stenosis. DWI and merged DWI and TiWI showed high signal intensity on DWI in the thickened left subclavian artery wall (Figure B). Because the spinal cord and the vertebra and rib bone marrow are usually the only structures that show increased signal of low diffusion in the thoracic area, high signal in the arterial tissue was easily visualized on DWI. Based on the clinical symptoms, physical examination, and MRI, the patient was diagnosed with Takayasu arteritis. Her symptoms gradually improved after treatment with oral prednisone $(30 \mathrm{mg} /$ day $)$, and the blood pressure in the left radial artery returned to $90 / 62 \mathrm{mmHg}$ at 5 weeks after the initiation of steroid therapy (blood pressure in right radial artery, $112 / 62 \mathrm{mmHg}$ ). MRI after 5 weeks of medical treatment showed reduction of arterial thickening and disappearance of the high signal intensity on DWI (Figure). Mean ADC in the arterial wall before and after the steroid therapy was $1.75 \pm 0.15 \times 10^{-3} \mathrm{~mm}^{2} / \mathrm{s}$ and $2.11 \pm 0.22 \times 10^{-3} \mathrm{~mm}^{2} / \mathrm{s}$, respectively.

The thickened arterial wall had high signal intensity on DWI, which reversed on steroid treatment. Kamper et al compared MRI, including DWI, in patients with chronic periaortitis before and after either prednisone or tamoxifen treatment. ${ }^{3}$ The periaortic fibrous tissue had high signal intensity on DWI, which resolved with treatment. Normal tissues, including spinal cord, bone marrow, and spleen, as well as neoplastic and inflammatory lesions, show low diffusion. Pathologic features of the acute phase of Takayasu arteritis are edema, adventitial perivascular lymphocytic infiltrate, patchy medial necrosis with multinucleated giant cells, and intimal hyperplasia. Because edema and inflammatory infiltrate are reversible, the impaired diffusion in the aorta may reflect inflammatory edema associated predominantly with adventitial and medial inflammation.

The arterial ADC before treatment were lower than after the treatment, similar to a previous study on chronic periaortitis. ${ }^{3}$ That study's ADC (before and after treatment, $0.89 \pm 0.23 \times 10^{-3} \mathrm{~mm}^{2} / \mathrm{s}$ and $1.27 \pm 0.22 \times 10^{-3} \mathrm{~mm}^{2} / \mathrm{s}$, respectively), however, were lower than in the present study. Possible reasons for the difference in the ADC may be due to anatomical and pathological differences, or differences in acquisition sequences, particularly the b-value and the sequence echo time. Therefore, the ADC of the lesion may be an objective marker for follow-up assessment in each patient, provided they are imaged on the same scanner with identical sequences.

Given that diffusion imaging is limited in spatial resolution, the diffusion images were merged with the TiWI to better localize the high signal intensity in the thickened subclavian artery wall, bone marrow and spinal cord. ${ }^{18} \mathrm{~F}$-FDG PET/CT is a reliable modality to assess regional inflammation, but is associated with radiation exposure, a concern for patients with Takayasu arteritis, who are usually young women. The gadolinium contrast agent for MRI carries a risk of allergic reaction. Therefore, the combination of diffusion-weighted MRI with other sequences may be a useful imaging modality to assess the vascular inflammation of Takayasu arteritis without radiation exposure or contrast agents. Because contrast-enhanced MRI failed to discriminate chronic active disease from inactive disease ${ }^{4}$ further study of DWI in the chronic phase of Takayasu arteritis is required.

\section{Disclosures}

This study was supported in part by Clinical Research from Miyazaki University Hospital and Intramural Research Fund (25-4-3) for Cardiovascular Diseases of the National Cerebral and Cardiovascular Center.

\section{References}

1. Kobayashi $Y$, Ishii $K$, Oda $K$, Nariai $T$, Tanaka $Y$, Ishiwata $K$, et al. Aortic wall inflammation due to Takayasu arteritis imaged with ${ }^{18}$ F-FDG PET coregistered with enhanced CT. J Nucl Med 2005; 46: 917-922.

2. Papa M, De Cobelli F, Baldissera E, Dagna L, Schiani E, Sabbadini M, et al. Takayasu arteritis: Intravascular contrast medium for MR angiography in the evaluation of disease activity. AJR Am J Roentgenol 2012; 198: W279-W284.

3. Kamper L, Haage P, Brandt AS, Piroth W, Abanador-Kamper $\mathrm{N}$, Roth S, et al. Diffusion-weighted MRI in the follow-up of chronic periaortitis. Br J Radiol 2015; 88: 20150145.

4. Kato Y, Terashima M, Ohigashi H, Tezuka D, Ashikaga T, Hirao K, et al. Vessel wall inflammation of Takayasu arteritis detected by contrast-enhanced magnetic resonance imaging: Association with disease distribution and activity. PLoS One 2015; 10: e 0145855 